\title{
The Value and Clinical Significance of Tumor Marker Detection in Cervical Cancer
}

\author{
Wei-Li Sun, Yong Shen (D, Yuan Yuan, Xiao-Jing Zhou, and Wei-Peng Li \\ Department of Nuclear Medicine, The First Affiliated Hospital of Bengbu Medical College, Bengbu, Anhui 233004, China \\ Correspondence should be addressed to Yong Shen; xgl1234321@163.com
}

Received 24 November 2020; Revised 22 December 2020; Accepted 4 February 2021; Published 24 February 2021

Academic Editor: Qinhu Zhang

Copyright (C) 2021 Wei-Li Sun et al. This is an open access article distributed under the Creative Commons Attribution License, which permits unrestricted use, distribution, and reproduction in any medium, provided the original work is properly cited.

\begin{abstract}
When it comes to cervical cancer, it is the most common malignancy in gynecology. This study aimed to investigate the concomitant status of miRNA-9-5p in cervical cancer and explore its potential mechanism for treating cervical cancer. The levels of miRNA-9-5p, CA125, CA199, and CEA expression were detected by RT-PCR, and the downstream target genes regulated by miRNA-9-5p were screened by the Venn map. Cytoscape was utilized to find the binding sites of the two genes, and luciferase reporter assay verified the direct regulation of miRNA-9-5p and CXCR4; the CCK-8 assay detected its regulation on cell proliferation, and the expression of miRNA-9-5p, CXCR4, PCNA, Ki67 mRNA, and proteins was detected by RT-PCR and western blot. The expression of miRNA-9-5p was decreased, while the levels of CA125, CA199, and CEA were increased in the model group. The database predicts that CXCR4 is a gene regulated by miRNA-9-5p. The luciferase reporter gene results indicated that miRNA-9-5p could directly regulate the expression of CXCR4 and miRNAs are detected by intracellular transfer inhibitors. In total, MiRNA-9-5p can be utilized as a biological marker for cervical cancer that may inhibit cancer cells' proliferation by inhibiting the expression of the CXCR4 gene and protein.
\end{abstract}

\section{Introduction}

When it comes to cervical cancer, this disease can be treated as the second most prevalent cancer in the world after breast cancer $[1,2]$. It ranked second in malignant tumors in women and is a disease that seriously endangers the health of women [3]. Treatment for early cervical cancer is primarily clinical surgery, but the onset of cervical cancer is hidden. Different screening methods and technologies make up more than half of the patients in the middle and advanced stages when they visit the clinic in different regions. The cure rate is relatively high for early cervical cancer $[2,4,5]$. Therefore, it is important to search for the cause and early diagnosis of cervical cancer and find new therapeutic targets.

MicroRNAs (miRNAs) are a class of endogenous small RNAs with a length of approximately 20-24 nucleotides that have a variety of important regulatory roles in the cells $[6,7]$. Each miRNA can have multiple target genes, and several miRNAs can also regulate the same gene. This complex regulatory network can regulate the expression of multiple genes through a single miRNA, or it can fine-tune the expression of a gene through a combination of several miRNAs [8]. It is speculated that miRNA regulates one-third of human genes. In this study, we found that miRNA-9-5p exhibits low expression in the serum of cervical cancer patients. Chen $\mathrm{H}$ found in osteoarthritis that miRNA-9-5p could regulate cell proliferation and apoptosis [9]. The level of serum CA125, CA199, and CEA showed that the expression was significantly higher in the model group than in the control group. Therefore, the above results indicate that miRNA-9-5p can be utilized as a biological marker of cervical cancer. At the same time, we also found that CA125, CA199, and CEA are highly expressed in cervical cancer, so it can be utilized as biological marker of cervical cancer.

Next, through various databases, we screened the downstream target genes regulated by miRNA-9-5p. We found that there are 330 target genes shared by five databases and regulated by miRNA-9-5p, in which CXCR4 is involved in the proliferation of cancer cells. Therefore, CXCR4 was utilized as a focus in the subsequent studies. 
The expression level of CXCR4 was significantly increased in various malignant tumor tissues. The expression of functional CXCR4 was found in the ovary, thyroid, mammary gland, stomach, lung, and other malignant tumors, indicating that CXCR4 plays a vital role in tumorigenesis and development. Therefore, our research was focused on the study of miRNA-9-5p regulation of CXCR4 in the treatment of cervical cancer.

In this paper, we verified the direct regulation of miRNA-9-5p and CXCR4 by the luciferase reporter gene. Subsequently, the miRNA-9-5p inhibitor and CXCR4 siRNA were transfected in Hela cells, and the cell viability was assessed by CCK-8 assay. MiRNA-9-5p can inhibit cell proliferation by inhibiting CXCR4 expression. To further verify its mechanism, we detected the expression of proliferative genes and proteins by RT-PCR and western blot and found that the level of CXCR4 mRNA in the model group was not significantly different from the control group. The expression of the protein was significantly increased, and miRNA-9-5p inhibitor significantly enhanced the expression of CXCR4. Simultaneously, with the low expression of miRNA-9-5p, the proliferation-related genes and proteins PCNA and Ki67 were highly expressed. Low expression of miRNA-9-5p promotes cell proliferation, and siRNA results indicate that blockade of the CXCR4 pathway reverses the ability of miRNA-9-5p to promote cell proliferation. The above results suggest that miRNA-9-5p promotes cell proliferation by regulating CXCR4 in cervical cancer.

\section{Materials and Methods}

2.1. The Patients. This study was carried out in the First Affiliated Hospital of Bengbu Medical College from June 2019 to June 2020. In this study, 32 patients with cervical cancer and 28 healthy people were admitted. The blood of patients and healthy people were collected, and the expression of miRNA-9-5p was detected by RT-PCR [10]. Levels of CA125, CA199, and CEA were detected by using the Roche c602 instrument and supporting reagents (electrochemiluminescence). The normal range for CA125 was $0-35 \mathrm{U} / \mathrm{mL}$, CA199 was $0-39 \mathrm{U} / \mathrm{mL}$, and CEA was $0-10 \mathrm{ng} / \mathrm{mL}$. If the level has exceeded the normal values, then it was considered positive.

2.2. Bioinformatics Analysis. The target genes of miR-9-5p were predicted by Targetscan [11] (http://www.targetscan. org/vert_72/), DIANA [12] (http://diana.imis.athenainnovation.gr/Diana Tools/index.php), miRmap [13] (https://mirmap.ezlab.org/), miRWalk [14] (http://zmf. umm.uni-heidelberg.de/apps/zmf/mirwalk/), and miRsystem (http://mirsystem.cgm.ntu.edu.tw/). The results were entered into the Draw Venn Diagram, and the results from the five databases were selected. Then, we get the sequence of miR-9-5p and the target gene and look for a combining site to identify the relationship between them. Then, we looked for the relative miRNAs of the target gene for cervical cancer and visualized by Cytoscape 3.6.1.
2.3. Cell Culture. Hela cells purchased from Shanghai Enzyme Research Biotechnology Co., Ltd., were divided into two groups: a control group and a model group. Cells were seeded, and the medium consisting of $10 \%$ fetal bovine serum (FBS; Gibco) and $1 \% \mathrm{PBS}$ at $37^{\circ} \mathrm{C}$ were added into the dish in the two groups, respectively. Moreover, in both groups, the expression of miRNA-9-5p was detected by RT-PCR [15].

2.4. Luciferase Reporter Assay. The relationship between miRNA-9-5p and CXCR4 was identified by luciferase reporter assay. CXCR4 was divided into two groups, including the wild-type CXCR4 (CXCR4 WT) and the mutant CXCR4 (CXCR4 MUT). In brief, CXCR4 WT or MUT 3'-UTR reporters together with miRNA-9-5p or negative control were cotransfected into the cells using Lipofectamine 2000 $[15,16]$. The plasmid (Promega) encoding luciferase was utilized to control the efficiency of transfection. Cells were lysed $24 \mathrm{~h}$ after transfection and tested for luciferase activity using the Dual-Luciferase Reporter Assay System (Promega), according to the manufacturer's instructions.

2.5. Inhibitor and siRNA Transfection. To identify the relationship between miRNA-9-5p and CXCR4, we transfected the miRNA-9-5p inhibitor into the cells using Lipofectamine $2000(5 \mu \mathrm{L})$ in a six-well plate. The cells were divided into three groups, the control group, model group, and inhibitor group. Cells were prepared for the following experiments.

2.6. CCK-8 Assay. In this study, $0.25 \%$ trypsin was added to the cells and placed at $37^{\circ} \mathrm{C}$ in an incubator with $5 \% \mathrm{CO}_{2}$. The cell counting plate was utilized for counting, and the cell concentration was adjusted to $1 \times 10^{4} / 200 \mu \mathrm{L}$. The prepared cells were seeded in 96-well plates at a volume of $200 \mu \mathrm{L}$ per well [17], and the cells were further incubated for about 8 hours. The experiment consisted of the following two groups, each with five duplicate wells. Control group: HHL-5 cell group; model group: Hela cell group.

After 24 hours, a 96-well plate was taken, and $20 \mu \mathrm{L}$ of the CCK8 reagent was added to each well. After 2 hours of incubation, a wavelength of $450 \mathrm{~nm}$ was selected, and the light absorption value of each well was measured on a microplate reader, and the results were recorded.

2.7. RT-PCR Analysis. The RT-PCR analysis was utilized to identify the expressions of miRNA-9-5p and CXCR4 and further demonstrate the correlation between two genes and cell proliferation. TRIZOL (Invitrogen) was utilized to extract RNA from cells with a confluency of about $85 \%$. And, microRNA reverse transcription was performed using bulge-loop TM miRNA reverse primer instead of Olige (dT) [18]. In this study, $\beta$-actin served as a control of CXCR4 and proliferating genes, while U6 snRNA (U6) served as a control of miRNA-9-5p. The primer sequences were as follows: CXCR4 F: $5^{\prime}$ AGGTGGTCTATGTTGGCGTCT-3', R: 5'-AGG ATGAGGATGACTGTGGTC-3'; PCNA F: 5'-TGATGAGGTCCTTGAGTG-3', R:5'-GAGTGGTCGTTG 
TCTTTC-3'; Ki67 F: 5'-CCTTCACCATCTTCCAGGAG-3', R: $5^{\prime}$-CCTGCTTCACCACCTTCTTG- $3^{\prime}$. Using $\beta$-actin as an internal reference, F: $5^{\prime}$-CTGGGACGACATGGAGAAAA- $3^{\prime}$, R: $5^{\prime}$-AAGGAAGGCTGGAAGAGTGC-3'; miRNA-9-5p F: $5^{\prime}$-GTGCAGGGT-CCGAGGT- ${ }^{\prime}, \quad$ R: $\quad 5^{\prime}$ GCGCTCTTTGGTTATCTAGC-3’.

2.8. Western Blot. Cells were lysed in a radioimmunoprecipitation assay buffer with the Protease Inhibitor Cocktail (Sigma), separated in sodium dodecyl sulfate-polyacrylamide gels, and transferred to a polyvinylidene fluoride membrane. The membrane was incubated with anti-CXCR4, PCNA, Ki67, and anti- $\beta$-actin (Abcam, Cambridge, MA, USA) at $4^{\circ} \mathrm{C}$ overnight, followed by incubation with horseradish peroxidase-conjugated secondary antibody for $1 \mathrm{~h}$. Bands were visualized with ECL [19].

2.9. Statistical Analysis. Data are presented as a mean\pm standard deviation. Statistical comparisons were carried out using the Student's $t$-test or one-way analysis of variance (ANOVA) followed by Tukey's multiple comparison test.

\section{Results}

3.1. Comparison of General Information in Each Group. In this study, the subjects treated in the hospital were included in the model group, 32 subjects, including 17 males and 15 females, aged between 36 and 75. The body's BMI value was 23.25 . The control group had 28 healthy subjects who passed the physical examination in the hospital, including 15 males, 13 females, aged between 20 and 77; body BMI was also within the normal range. There was no statistical difference between the general information of the model combination control group, and it was comparable. See Table 1 for details.

3.2. 330 Target Genes Regulated by miRNA-9-5p and miRNA-9-5p Have Low Expression in Cervical Cancer. Through the database search, the target genes regulated by miRNA-9-5p were predicted from five databases, including TargetScan, DIANA, miRmap, miRWalk, and miRsystem. Then, 330 target genes that are regulated by miRNA-9-5p and coexist in five databases were selected and represented as Venn maps (Figure 1(a)). Among them, CXCR4 was abnormally expressed in various malignant tumor tissues and cell lines such as hematological tumors, breast tumors, and prostate tumors, indicating that CXCR4 plays an important role in tumorigenesis and development, and the binding sites of two genes were predicted by TargetScan database. Visualization via Cytoscape showed that the relative miRNAs which regulates CXCR4 were miRNA-9-5p, miRNA-150-5p, miRNA-146a-5p, miRNA-126-3p, and so on (Figure 1(b)).

To demonstrate the expression of miRNA-9-5p in the serum of patients with cervical cancer, we collected serum from the patients of both control and model groups. RT-PCR results showed that the expression of miRNA-9-5p in the model group was significantly lower than that in the control group (Figure 1(c)). The serum level of CA125, CA199, and CEA showed that the expression in the model group was significantly higher than that in the control group. The above results indicate that they may serve as a biological marker for cervical cancer.

\subsection{MiRNA-9-5p Could Regulate the Expression of CXCR4} and Inhibit the Proliferation of Hela Cells. To validate that the function of miRNA-9-5p directly regulates the expression of CXCR4, we found a site between miRNA-9-5p and CXCR4 to identify the mechanism. The binding sites between miRNA-9-5p and CXCR4 were mutated to determine the binding ability of two genes. The results of the luciferase reporter assay showed that the miRNA-9-5p could bind to CXCR4-WT and decrease the fluorescence intensity, but the mutated CXCR4 would not bind to miRNA-9-5p, and the fluorescence intensity would not be affected by the amount of miRNA-9-5p expression (Figure 2(a)). These results indicate that CXCR4 is a target gene that is directly regulated by miRNA-9-5p.

To identify the effects of miRNA-9-5p and CXCR4 on the proliferation of cervical cancer cells, miRNA-9-5p inhibitor and CXCR4 siRNA were transfected. The results of CCK-8 showed that the cell viability of the model group was increased compared to the control group, and the miRNA-9-5p inhibitor significantly increased the cell viability of the hepatoma cells. Transfection of CXCR4 siRNA resulted in a decrease in cell proliferation ability (Figure 2(b)). Low expression of miRNA-9-5p may promote cell proliferation by regulating CXCR4 expression.

3.4. MiRNA-9-5p Inhibitor Could Increase the Expression of CXCR4. Low expression of miRNA-9-5p can promote cell proliferation, but the mechanism of specific regulation of cell proliferation is still unclear. To identify the influence of miRNA-9-5p on the expression of CXCR4, the miRNA-9-5p inhibitor and CXCR4 siRNA were transfected into the Hela cells. Therefore, in every group, including CXCR4, PCNA, and Ki67, this experiment detected miRNA-9-5p-related gene expression by RT-PCR (Figure 3 ).

The results showed that the expression of CXCR4 was significantly increased in the model group compared to the control group $(P<0.01)$. The inhibitor significantly increased the expression of CXCR4, and CXCR4 siRNA interfered with the expression of CXCR4. These findings indicated that there was a negative correlation between miRNA-9-5p and CXCR4. The results from previous studies showed that the expression changes of miRNA-9-5p and CXCR4 could affect the proliferation of cells [9]. Therefore, we also examined the expression of PCNA and Ki67. The results showed that the proliferation of Hela cells was significantly enhanced compared to the control group. Due to the low expression of miRNA-9-5p, the inhibitor group significantly increased the proliferation of cancer cells, indicating that the low expression of miRNA-9-5p may lead to rapid cell proliferation; the proliferation ability of siRNA group cells is significantly higher than that of the model group and the inhibitor group. 
TABLE 1: Comparison of general conditions between the two groups.

\begin{tabular}{lcc}
\hline Project & Model group & Control group \\
\hline Number & 32 & 28 \\
Male/Female & $17 / 15$ & $15 / 13$ \\
Ages & $36-75$ & $20-77$ \\
BMI $(x \pm s), \mathrm{kg} / \mathrm{m}^{2}$ & $23.25 \pm 1.17$ & $22.32 \pm 1.57$ \\
\hline
\end{tabular}

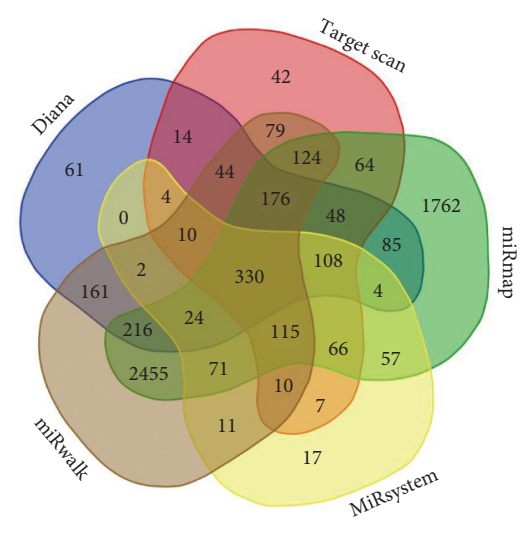

(a)

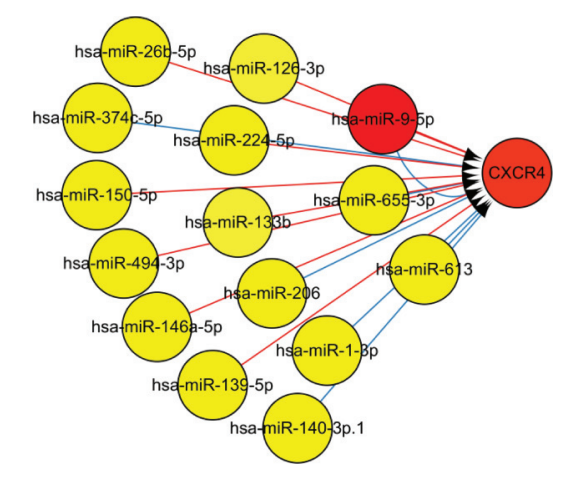

(b)

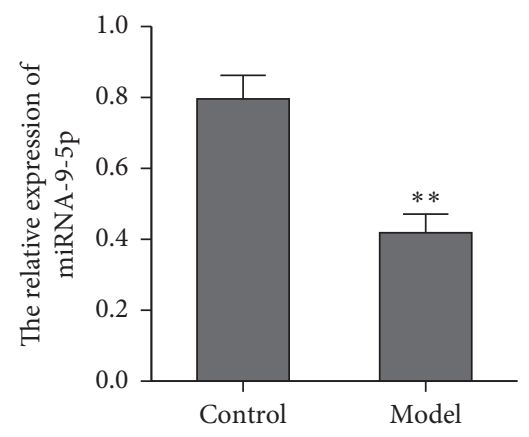

(c)

FIgURE 1: The target genes regulated by miRNA-9-5p and the expression of miRNA-9-5p in cervical cancer patients. (a) The target genes regulated by miRNA-9-5p were detected among databases; (b) The CXCR4 relative miRNAs were predicted by Cytoscape software. (c) The expression of miRNA-9-5p in patients of cervical cancer. ${ }^{* *} P<0.01 v s$. Control group.

The above results indicate that low expression of miRNA-9-5p regulates cell proliferation and may be involved in the regulation of CXCR4 gene expression.

3.5. MiRNA-9-5p Inhibit the Proliferation of Hela Cells by Regulating CXCR4. In the above, we verified the mechanism of miRNA-9-5p inhibiting cell proliferation by regulating CXCR4 from the gene level, and then, western blot was also utilized to verify the effect of miRNA-9-5p on proliferation-related protein and CXCR4 protein expression (Figure 4). The CXCR4 results showed that the expression of CXCR4 protein was significantly increased in the model group compared to the control group. The inhibitor group resulted in a decrease in the expression of miRNA-9-5p and a decrease in the expression of CXCR4 protein. SiRNA also caused a decrease in the expression of CXCR4 protein. The results of PCNA and Ki67 protein were consistent with the results of gene detection. The above findings indicate that the low expression of miRNA-9-5p can be utilized as a biological marker of cervical cancer, and its low expression can promote cell proliferation by up-regulating CXCR4 protein expression. Therefore, treatment of cervical cancer can be achieved by activating the miRNA-9-5p-consistent CXCR4 expression to inhibit cancer cell proliferation.

\section{Discussion}

Cervical cancer is the second most common reproductive tract malignancy in women $[20,21]$. Data show that China has about 130,000 new cervical cancer cases every year, accounting for one-third of new cases worldwide, and about 80,000 women die each year. Cervical cancer is also the second most common cancer among women aged 15 to 44 in China [22]. Globally, a new case is detected every minute, and a woman dies of cervical cancer every two minutes. In the early stages, because traditional therapies, such as surgery, chemotherapy, and radiotherapy, have unsatisfactory results and poor prognosis, cervical cancer has a low cure 


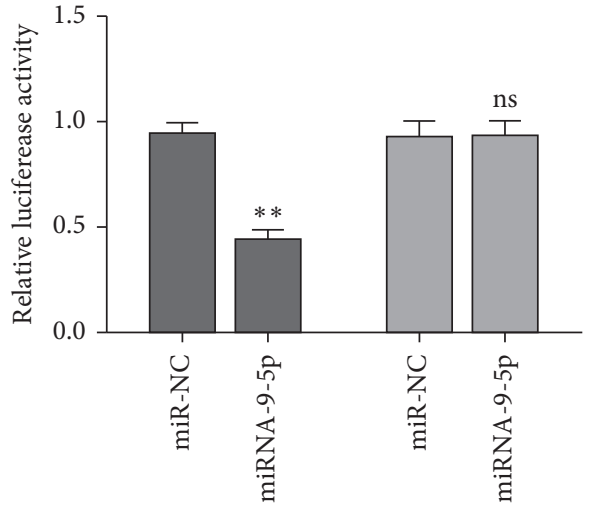

CXCR4-WT

CXCR4-MUT

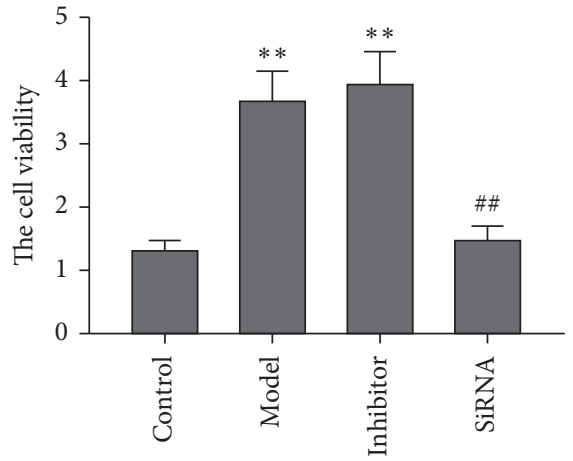

(b)

FIGURE 2: The luciferase report assay and cell viability. (a) The over-expressed miRNA-9-5p was able to bind CXCR4-WT, and the fluorescence intensity was weakened, and the difference was significant $(P<0.01)$. In combination with CXCR4-MUT, there is no difference in fluorescence intensity. (b) The low expression of miRNA-9-5p could increase the cell viability in cervical cancer. ${ }^{* *} P<0.01 v s$. Control group; ${ }^{\#} P<0.001$ vs. Model group.

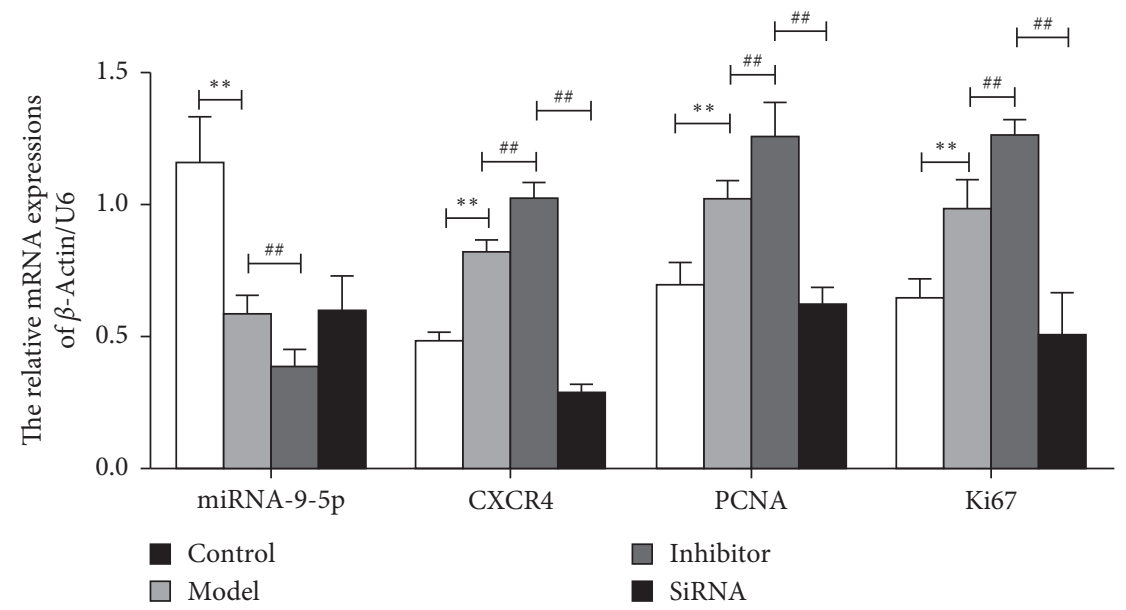

FIGURE 3: Expression of miRNA-9-5p and CXCR4 in cervical cancer. RT-PCR analyzed the expression of miRNA-9-5p, CXCR4, PCNA, and Ki67; The expression levels were semi-quantified by densitometric measurements, normalized with U6/ $\beta$-actin as an internal control. ${ }^{* *} P<0.01$ vs. CON; ${ }^{\# \#} P<0.001$ vs. Model.
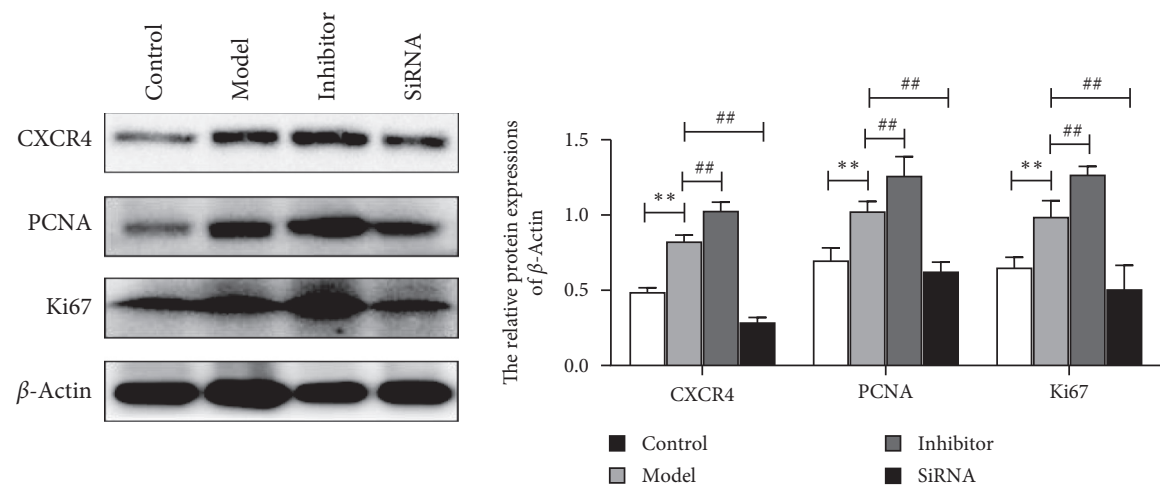

FIGURE 4: Expression of CXCR4, PCNA, and Ki67 proteins in cervical cancer. The expression of CXCR4, PCNA, and Ki67 proteins were analyzed by western blot, and the expression levels were semi-quantified by densitometric measurements, normalized with $\beta$-actin as the internal control. ${ }^{* *} P<0.01$ vs. CON; ${ }^{\# \#} P<0.001$ vs. Model. 
rate. Therefore, the search for the cause of cervical cancer, the early diagnosis of cervical cancer, and new therapeutic targets are particularly important.

Studies have shown that a variety of factors can lead to recurrence and metastasis of cervical cancer. miRNAs are widely present in eukaryotic cells and have 22 to $23 \mathrm{nu}-$ cleotides in length [23-25]. They are highly conserved, contribute to cell proliferation, differentiation, and apoptosis, and play an important role in developing malignant tumors. miRNAs account for only $1 \%$ to $3 \%$ of total genes but can regulate more than $30 \%$ of gene expression. Approximately $50 \%$ of tumor tissues can detect miRNA expression, and the results are reproducible, indicating that miRNAs may be closely related to the development of tumors. The expression of miRNAs is different in different tumors, such as the up-regulation of miRNA-10b and miRNA-21 in tumor tissues [26-28]. Targeting the expression of mRNA by miRNA is a novel molecular targeted therapy. The binding of miRNA to mRNA has complementary features, but it is not completely complementary. By predicting whether miRNA and mRNA are bound by related software, specific targets for antitumor therapy can be found, and a variety of software with higher predictive value can be utilized to simultaneously predict and analyze possible outcomes. In this article, the target genes regulated by miRNA-9-5p were predicted by TargetScan, DIANA, miRmap, miRWalk, and miRsystem databases, and we found that the five databases coexist, and the gene associated with cervical cancer cell proliferation was the CXCR4 gene, that we visualized through the Venn map.

Experimental studies have shown that CXCR4 can promote tumor progression through direct and indirect mechanisms. It mainly involves the following pathways: first, CXCR4 can induce tumor cells to metastasize to CXCL12positive tissues and organs $[29,30]$ and thus cause the entry of tumor cells into the bone marrow cell, which allows the tumor cells to escape from the body's immune killing effect, which is beneficial to tumor survival and growth. Secondly, the interstitial cell-derived CXCL12 itself stimulates the survival and growth of neoplastic cells in a paracrine manner. Third, CXCL12 promotes tumor angiogenesis by attracting endothelial cells to the tumor microenvironment [31]. Moreover, data were also suggesting that increased expression of functional CXCR4 is a precursor to many tumors. Taken together, these findings suggest that CXCR4 plays an important role in the spread and progression of a variety of tumors. Therefore, CXCR4 can be an effective factor in the treatment of tumors.

In this study, the luciferase reporter assay was utilized to verify the regulatory relationship between miRNA-9-5p and CXCR4. The results show that miRNA-9-5p has a direct regulatory effect on CXCR4. Subsequently, we transfected miRNA-9-5p inhibitor and CXCR4 siRNA in Hela cells and found that miRNA-9-5p can be inhibited by the CCK- 8 assay. CXCR4 expression also inhibited cell proliferation. To further verify this mechanism, we quantified the expression of proliferation-related genes and proteins by RT-PCR and western blot. The results showed that the expression of the CXCR4 gene and protein was significantly increased in the model group compared to the control group. The miRNA-9-5p inhibitor can significantly enhance the expression of CXCR4, and with the low expression of miRNA-9-5p, the proliferation-related genes and proteins, PCNA and Ki67 were highly expressed. The above results indicate that miRNA-9-5p was low. The expression can promote cell proliferation, and siRNA results indicated that blocking of the CXCR4 pathway reverses the ability of miRNA-9-5p to promote cell proliferation. The above results indicate that miRNA-9-5p promotes cell proliferation in cervical cancer by regulating the CXCR4. The above results are consistent with the aim of this article. miRNA-9-5p can therefore be utilized as a biological marker for cervical cancer to prevent cervical cancer and is of great significance for treating cervical cancer.

\section{Data Availability}

All data used to support the study are included within this article.

\section{Conflicts of Interest}

The authors declare that they have no conflicts of interest regarding the publication of this paper.

\section{Acknowledgments}

This work was supported by the Natural Science Fund Project of Bengbu Medical College (Grant no. BYKY2019026ZD) and Development of Science and Technology Fund Project of Bengbu Medical College (Grant no. BYKF1795).

\section{References}

[1] X. Gao, G. Chen, H. Cai, X. Wang, K. Song, and L. Liu, "Aberrantly enhanced melanoma-associated antigen (MAGE)-A3 expression facilitates cervical cancer cell proliferation and metastasis via actuating Wnt signaling pathway," Biomed Pharmacother, vol. 122, Article ID 109710, 2020.

[2] P. Huang, B. Qi, H. Yao, L. Zhang, Y. Li, and Q. Li, "Circular RNA cSMARCA5 regulates the progression of cervical cancer by acting as a microRNA432 sponge," Molecular Medicine Reports, vol. 21, no. 3, 2020.

[3] W. Bao, Y.-H. Huang, and Y. H. Chen, "MSIT: malonylation sites identification tree," Current Bioinformatics, vol. 15, no. 1, pp. 59-67, 2020.

[4] A. Christodoulou, J. Ajzajian, D. Su, H. Wang, Z. Roupa, and P. A. Farazi, "Awareness of human papilloma virus and cervical cancer prevention among Cypriot female healthcare workers," Ecancermedicalscience, vol. 13, p. 978, 2019.

[5] B. Wenzheng and Y. Bin, "LAIPT: lysine acetylation site identification with polynomial tree," International Journal of Molecular Sciences, vol. 20, no. 1, p. 113, 2018.

[6] J. Pordzik, D. Jakubik, J. Jarosz-Popek, Z. Wicik, C. Eyileten, and S. De Rosa, "Significance of circulating microRNAs in diabetes mellitus type 2 and platelet reactivity: bioinformatic analysis and review," Cardiovasc Diabetol, vol. 18, no. 1, p.113, 2019.

[7] W. Bao, D. B. Yang, and Y. Z. ZhouLi, "CMSENN: computational modification sites with ensemble neural network," 
Chemometrics and Intelligent Laboratory Systems, vol. 185, pp. 65-72, 2019.

[8] W. Bao, D.-S. Yang, B. Liu, Q. Wang, Y.-H. Chen, and R. Bao, "IMKPse: identification of protein malonylation sites by the key features into general PseAAC," IEEE Access, vol. 7, pp. 54073-54083, 2019.

[9] W. Bao, Y. Yuan, K. Han, B. Honig, and D.-S. Huang, "Mutlifeatures prediction of protein translational modification sites," IEEE/ACM Transactions on Computational Biology and Bioinformatics, vol. 15, no. 5, pp. 1453-1460, 2018.

[10] L. Nandi, L. Pezzi, I. Corcostegui, A. Dubot-Peres, A. Falchi, and $\mathrm{X}$. de Lamballerie, "Development and evaluation of a duo chikungunya virus real-time RT-PCR assay targeting two regions within the genome," Viruses, vol. 11, no. 8, 2019.

[11] D. Mon-Lopez and C. M. Tejero-Gonzalez, "Validity and reliability of the targetScan ISSF Pistol \& Rifle application for measuring shooting performance," Scandinavian Journal of Medicine \& Science in Sports, vol. 29, no. 11, pp. 1707-1712, 2019.

[12] W. Bao, Y. D. Wang, and Y. Chen, "Classification of protein structure classes on flexible neutral tree," IEEE/ACM Transactions on Computational Biology and Bioinformatics, vol. 14, no. 5, pp. 1122-1133, 2017.

[13] E. A. Goossens, M. R. De Vries, K. H. Simons, P. Hein, P. H. Quax, and A. Y. Nossent, "miRMap: profiling $14 \mathrm{q} 32$ microRNA expression and DNA methylation throughout the human vasculature," Frontiers in Cardiovascular Medicine, vol. 6, p. 133, 2019.

[14] C. Sticht, C. De La Torre, A. Parveen, and N. Gretz, "miRWalk: an online resource for prediction of microRNA binding sites," PLoS One, vol. 13, no. 10, Article ID e0206239, 2018.

[15] F. Anower-E-Khuda, G. Singh, Y. Deng, P. L. S. M. Gordts, and J. D. Esko, "Triglyceride-rich lipoprotein binding and uptake by heparan sulfate proteoglycan receptors in a CRISPR/Cas9 library of Hep3B mutants," Glycobiology, vol. 29, no. 8, pp. 582-592, 2019.

[16] H. Unal, "Luciferase reporter assay for unlocking ligandmediated signaling of GPCRs," Methods in Cell Biology, vol. 149, pp. 19-30, 2019.

[17] X. Wan, C. Zheng, and L. Dong, "Inhibition of CTRP6 prevented survival and migration in hepatocellular carcinoma through inactivating the AKT signaling pathway," Journal of Cellular Biochemistry, vol. 120, no. 10, pp. 17059-17066, 2019.

[18] K. T. Que, Y. Zhou, Y. You, Z. Zhang, X. P. Zhao, and J. P. Gong, "MicroRNA-31-5p regulates chemosensitivity by preventing the nuclear location of PARP1 in hepatocellular carcinoma," Journal of Experimental \& Clinical Cancer Research, vol. 37, no. 1, p. 268, 2018.

[19] W. Bao and D. Huang, S. Yuan, C. A. Z. Huang, and D. S. Huang, Pupylation sites prediction with ensemble classification model," International Journal of Data Mining and Bioinformatics, vol. 18, no. 2, pp. 91-104, 2017.

[20] J. L. Chen, M. C. Wang, Y. S. Huang, C. Y. Huang, C. K. Pan, and C. Y. Hsu, "Extended-field bone marrow sparing radiotherapy for primary chemoradiotherapy in cervical cancer patients with para-aortic lymphadenopathy: volumetricmodulated arc therapy versus helical tomotherapy," Journal of X-Ray Science and Technology, vol. 28, no. 1, pp. 111-124, 2020.

[21] X. Jiang, J. Li, Y. Kan et al., "MRI based radiomics approach with deep learning for prediction of vessel invasion in earlystage cervical cancer," IEEE/ACM Transactions on Computational Biology and Bioinformatics, p. 1, 2020.
[22] X. Peng, Y. Zhang, J. Gao, and C. Cai, "MiR-1258 promotes the apoptosis of cervical cancer cells by regulating the E2F1/ P53 signaling pathway," Experimental and Molecular $\mathrm{Pa}$ thology, vol. 114, Article ID 104368, 2020.

[23] D. G. Sur, M. Colceriu, G. Sur et al., "MiRNAs roles in the diagnosis, prognosis and treatment of colorectal cancer," Expert Review of Proteomics, vol. 16, no. 10, pp. 851-856, 2019.

[24] S. Ning, H. Liu, B. Gao et al., "miR-155, miR-96 and miR-99a as potential diagnostic and prognostic tools for the clinical management of hepatocellular carcinoma," Oncology Letters, vol. 18, no. 3, pp. 3381-3387, 2019.

[25] Q. Chen, Z. Chen, S. Cao et al., "Role of CircRNAs_100395 in proliferation and metastases of liver cancer," Medical Science Monitor, vol. 25, pp. 6181-6192, 2019.

[26] H. Li, J. Fan, L. Fan et al., "MiRNA-10b reciprocally stimulates osteogenesis and inhibits adipogenesis partly through the TGF- $\beta$ /SMAD2 signaling pathway," Aging and Disease, vol. 9, no. 6, pp. 1058-1073, 2018.

[27] L. Li and D. Jiang, "Hypoxia-responsive miRNA-21-5p inhibits Runx2 suppression by targeting SMAD7 in MC3T3-E1 cells," Journal of Cellular Biochemistry, vol. 120, no. 10, pp. 16867-16875, 2019.

[28] H. F. Hetta, A. M. Zahran, R. I. El-Mahdy et al., “Assessment of circulating miRNA-17 and miRNA-222 expression profiles as non-invasive biomarkers in egyptian patients with nonsmall-cell lung cancer," Asian Pacific Journal of Cancer Prevention, vol. 20, no. 6, pp. 1927-1933, 2019.

[29] L. Chen, J. Ouyang, K. Wienand et al., "CXCR4 upregulation is an indicator of sensitivity to B-cell receptor/PI3K blockade and a potential resistance mechanism in B-cell receptor-dependent diffuse large B-cell lymphomas," Haematologica, vol. 105, no. 5, pp. 1361-1368, 2019.

[30] T. Manoochehrabadi, Z. Sharifi, and F. Yari, "Role of plateletderived microparticles in transfer of the chemokine receptor CXCR4 to CXCR4-negative cells," Medical Journal of the Islamic Republic of Iran, vol. 33, p. 55, 2019.

[31] A. Ruiz, L. Ruiz, M. Colón-Caraballo et al., "Pharmacological blockage of the CXCR4-CXCL12 axis in endometriosis leads to contrasting effects in proliferation, migration, and invasion†," Biology of Reproduction, vol. 98, no. 1, pp. 4-14, 2018. 